\title{
Spot urinary vitamin $C$ and urinary potassium: novel biomarkers of fruit and vegetable consumption?
}

\author{
A. J. McGrath, L. L. Hamill, G. Graham, S. E. C. M. Gilchrist, I. S. Young, M. C. McKinley \\ and J. V. Woodside \\ Centre for Public Health, Institute of Clinical Science B, Grosvenor Road, Belfast, Northern Ireland, BT12 6BJ, UK
}

Plasma vitamin C concentration plateaus at higher levels of FV intake ${ }^{(1)}$, therefore spot urinary vitamin $\mathrm{C}$ may be a better FV intake biomarker. Potassium $(\mathrm{K})$ is found widely in FV and excreted in urine ${ }^{(2)}$. Thus, using data from a randomised FV study, we explored the use of spot urinary vitamin $\mathrm{C}$ and $\mathrm{K}$ as potential novel biomarkers of $\mathrm{FV}$ intake.

Participants were aged 40-65 years and hypertensive (blood pressure of 140-179/90-109 mmHg). Following a one portion FV/day four-week run-in-period, participants were randomised to consume 1, 3 or 6 portions FV/day for eight-weeks. Fasting plasma, spot and 24-hour urine samples were collected pre- and post-intervention. Plasma vitamin $\mathrm{C}$ was measured using a fluorimetric method on an automated Cobas Fara centrifugal analyser. Urinary vitamin C was measured on a BMG FLUOstar Optima plate reader. Urinary $\mathrm{K}$ was measured on the ion selective electrode module of a Cobas analyser.

A total of 117 subjects completed the 12-week study. Across the intervention groups plasma vitamin $\mathrm{C}$ increased, but plateaued between 3 and 6 portions/d. In contrast, spot and 24-hour urinary vitamin C increased as FV intake increased. No statistically significant differences were found between the three groups in spot and 24-hour urinary $\mathrm{K}$ excretion.

Urinary vitamin $\mathrm{C}$, but not urinary $\mathrm{K}$ excretion, may be a potential biomarker of $\mathrm{FV}$ intake.

\begin{tabular}{|c|c|c|c|c|c|c|}
\hline & & & 1 portions/d $\left(\mathrm{n}_{\max }=29\right)$ & 3 portions/d $\left(\mathrm{n}_{\max }=38\right)$ & 6 portions/d $\left(\mathrm{n}_{\max }=38\right)$ & $p$-value \\
\hline \multirow{4}{*}{$\begin{array}{l}\text { Plasma } \\
\text { vitamin } C \\
(\mu \mathrm{mol} / 1)^{1}\end{array}$} & Baseline & Mean & $23 \cdot 7$ & $25 \cdot 7$ & $27 \cdot 9$ & 0.62 \\
\hline & & IQR & $15 \cdot 5,34 \cdot 3$ & $20 \cdot 9,36 \cdot 7$ & $19 \cdot 2,45 \cdot 0$ & \\
\hline & Change 8 wk & Mean & 1.09 & & & 0.06 \\
\hline & & $95 \% \mathrm{CI}$ & $0 \cdot 90,1 \cdot 32$ & $1 \cdot 20,1.91$ & $1 \cdot 26,1 \cdot 83$ & \\
\hline \multirow{4}{*}{$\begin{array}{l}\text { Spot urinary } \\
\text { vitamin C } \\
(\mu \mathrm{mol} / 1)^{1}\end{array}$} & Baseline & Mean & $141 \cdot 17$ & $144 \cdot 95$ & $107 \cdot 98$ & $0 \cdot 193$ \\
\hline & & & $97 \cdot 55,211 \cdot 60$ & $90 \cdot 12,194 \cdot 65$ & $68 \cdot 41,182 \cdot 86$ & \\
\hline & Change 8 wk & Mean & $0.93^{\mathrm{a}}$ & $1 \cdot 22^{\mathrm{ab}}$ & $1 \cdot 64^{\mathrm{b}}$ & $0 \cdot 001$ \\
\hline & & $95 \% \mathrm{CI}$ & $0 \cdot 78,1 \cdot 10$ & $0.97,1.52$ & $1 \cdot 24,2 \cdot 19$ & \\
\hline \multirow{4}{*}{$\begin{array}{l}\text { 24-hour urinary } \\
\text { vitamin } C \\
(\mu \mathrm{mol} / 1)^{1}\end{array}$} & Baseline & Mean & 143.97 & $102 \cdot 56$ & $107 \cdot 13$ & $0 \cdot 112$ \\
\hline & & & $109 \cdot 35,178 \cdot 72$ & $78 \cdot 36,145 \cdot 36$ & $71 \cdot 45,180 \cdot 03$ & \\
\hline & Change $8 \mathrm{wk}$ & Mean & $0.95^{\mathrm{a}}$ & $1 \cdot 17^{\mathrm{a}}$ & $2 \cdot 23^{\mathrm{b}}$ & $<0 \cdot 001$ \\
\hline & & $95 \% \mathrm{CI}$ & $0 \cdot 81,1 \cdot 12$ & $0 \cdot 91,1 \cdot 50$ & $1.63,3.03$ & \\
\hline \multirow{4}{*}{$\begin{array}{l}\text { Spot urinary } \\
\text { potassium } \\
(\mathrm{mmol} / \mathrm{l})^{2}\end{array}$} & Baseline & Mean & $67 \cdot 2$ & $63 \cdot 5$ & $61 \cdot 7$ & 0.71 \\
\hline & & & $40 \cdot 2$ & $35 \cdot 4$ & $43 \cdot 0$ & \\
\hline & Change 8 wk & Mean & $-16 \cdot 1$ & & $-2 \cdot 1$ & 0.428 \\
\hline & & $95 \% \mathrm{CI}$ & $-30 \cdot 2,-1 \cdot 9$ & $-17 \cdot 5,23 \cdot 1$ & $-20 \cdot 3,16 \cdot 0$ & \\
\hline \multirow{4}{*}{$\begin{array}{l}\text { 24-hour urinary } \\
\text { potassium } \\
(\mathrm{mmol} / \mathrm{l})^{2}\end{array}$} & Baseline & Mean & $36 \cdot 6$ & $42 \cdot 6$ & $38 \cdot 5$ & $0 \cdot 901$ \\
\hline & & & $17 \cdot 1$ & $20 \cdot 7$ & $21 \cdot 2$ & \\
\hline & Change 8 wk & Mean & $-5 \cdot 1$ & $-3 \cdot 7$ & $-1 \cdot 2$ & 0.604 \\
\hline & & $95 \% \mathrm{CI}$ & $-13 \cdot 6,3 \cdot 4$ & $-17 \cdot 8,10 \cdot 5$ & $-10 \cdot 3,7 \cdot 9$ & \\
\hline
\end{tabular}

${ }^{1}$ Variables were logarithmically transformed. All baseline values are geometric mean (IQR), and all change values are geometric mean (95\% CIs) of the ratio of the week 8 to baseline value. ${ }^{2}$ All baseline values are mean (SD), and all changes mean $(95 \% \mathrm{CI})$. Changes were calculated as week 8 - baseline; changes were compared between groups using one way analysis of variance with a test for linear trend. Superscripted letters indicate homogeneous subsets.

1. Jenab M, Slimani N, Bictash M, Ferrari P, Bingham SA (2009) Hum Genet 125, 507-525.

2. Bingham SA (2002) Public Health Nutr 5(6A), 821-827. 